\title{
Quantum degeneracy and phase space density in superradiant semiconductor heterostructures at room temperature
}

\author{
PETER P. VASIL'EV ${ }^{1,2(a)}$ and RICHARD V. PENTY ${ }^{1}$ \\ ${ }^{1}$ Centre for Photonic Systems, University of Cambridge, 9 JJ Thomson Avenue, Cambridge, CB3 OFA United Kingdom \\ ${ }^{2}$ Quantum Radiophysics Department, PN Lebedev Physical Institute, 53 Leninsky Prospect, Moscow 119991, Russia \\ PACS 42.50.Fx - Cooperative phenomena in quantum optical systems \\ PACS 71.35.Lk - Collective effects (Bose effects, phase space filling, and excitonic phase transitions) \\ PACS 67.85.Jk - Other Bose-Einstein condensation phenomena
}

\begin{abstract}
We investigate the fulfilment of the quantum degeneracy criterium in bulk GaAs/AlGaAs heterostructures operated under the superradiant emission generation at room temperature. The e-h density has been evaluated by analysing both spontaneous window emission from above the samples and amplified spontaneous emission radiated from their edges. The maximum experimental value of the phase-space density is 1.31 at $\mathrm{T}=300 \mathrm{~K}$. It is supposed that resonant photons with energies at the band gap establish coherence within the e-h ensemble and facilitate the build-up of a macroscopic wavepacket from overlapped wavefunctions of collectively paired electrons and holes. As a result, the photon-mediated 'stimulated' condensation of e-h pairs in phase space can take place at room temperature
\end{abstract}

Introduction. - Bose-Einstein condensation (BEC) has been attracted a great deal of interest for many years especially since remarkable series of experiments on vapours of rubidium and sodium in which the atoms were confined in magnetic traps and cooled down to extremely low temperatures $[1,2]$. The condition for this to happen is that the phase-space density $\mathrm{n} \Lambda^{3}$ must be greater than unity, $\mathrm{n} \Lambda^{3}>1$ (the quantum degeneracy) [3]. Here, $\mathrm{n}$ is the particle density and $\Lambda$ is the de Broglie thermal wavelength. The latter characterises the region which the particle wavefunction occupies. When $\Lambda$ is much smaller than the interparticle spacing, quantum effects will be negligible, and the particles will behave classically. In the opposite case, quantum effects will be important. On the other words, it is required for the condensation that the de Broglie wavelength $\Lambda$ of each particle must be large enough to overlap with its neighbour. As the phase-space density increases, the particle waves begin to interfere with each other, a macroscopic coherent wavepacket and phase coherence are then formed in the system, and condensation occurs provided the particles are bosons.

Much effort has been devoted to finding a solid-state system with composite bosons, i.e. electron-hole pairs in semiconductors, which BEC can take place [4,5]. For instance, the Coulomb attraction ensures the formation of excitons, which may condense and obey the Bose statistics at low densities and low enough temperatures. In the large-density case, collectively paired electrons and holes may form a BCSlike state that is quite similar to the ensemble of Cooper pairs in a superconductor. The possibility of condensation in such systems was first discussed by Keldysh and co-workers many years ago [6]. Since then, the e-h condensation has been widely studied both theoretically and experimentally [7-10].

The de Broglie thermal wavelength is given by

$$
\Lambda=\sqrt{\frac{2 \pi \hbar^{2}}{M k T}}
$$

where $\mathrm{M}$ is a sum of the masses of an electron and a hole in a semiconductor and $\mathrm{T}$ is the temperature. According to Eq. (1), the quantum degeneracy can be achieved and the phase-space density can be increased either by decreasing the temperature or by increasing the particle density. The first way is a mainstream approach for the achievement of the condensation. We use the second method. Indeed, the de Broglie wavelength of a bound electron-heavy hole pair in GaAs is $5.9 \times 10^{-7} \mathrm{~cm}$ at room temperature. The exciton Bohr radius is around $1.4 \times 10^{-6} \mathrm{~cm}$ which is much larger than $\Lambda$. Figure 1 illustrates an ensemble of e-h pairs at low $n \Lambda^{3}<<1$ (a) and high $n \Lambda^{3}>1$ (b) phasespace densities. Fig. 1(a) corresponds to almost most of the semiconductors at room temperature. For instance, typical operating densities in semiconductor lasers are in the range of $(1.5-2.0) \times 10^{18} \mathrm{~cm}^{-3}$ [11], which corresponds to the phasespace density of $0.29-0.39$. If the e-h density $\mathrm{n}$ is high enough, the individual de Broglie wavelengths overlap and can form a macroscopic wavepacket (Fig. 1(b)) 


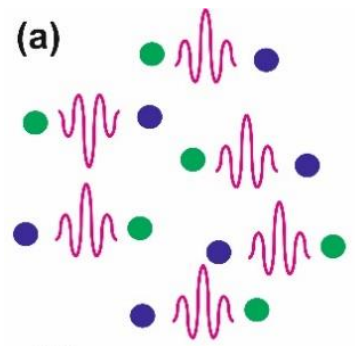

(b)

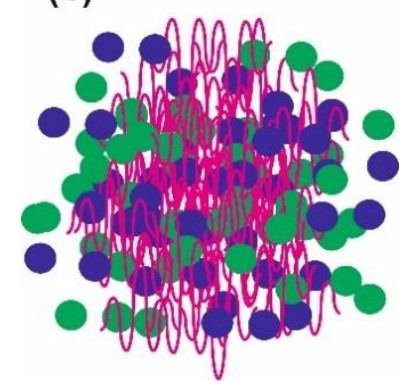

Fig. 1: Bound electrons (green circle) and holes (blue circle) and their wave functions (red) in case of a small (a) and large (b) density at the same temperature.

Our previous experimental and theoretical studies have demonstrated that the photon-mediated collective pairing in a high-density e-h ensemble and e-h non-equilibrium condensation in phase space has resulted in the formation of a short-living coherent e-h BCS-like state [9,12-14]. Besides, the formation of off-diagonal long-range order in this system has been observed experimentally [14]. The conditions and proposed mechanism of e-h condensation in phase space at room temperature have also been described [15]. The nonequilibrium e-h BCS-like state recombines in a superradiant (SR) manner. Moreover, the observed SR emission exhibits severe deviations from the Poissonian statistics of the photons, the Wigner function shows large areas of negative values, a characteristic sign of non-classicality, demonstrating the quantum nature of the generated light [16].

In this work, we investigate the fulfilment of the quantum degeneracy criterion and estimate the phase-space density in $\mathrm{GaAs} / \mathrm{AlGaAs}$ heterostructures during the superradiant emission generation. The e-h density is estimated using the band-gap shrinkage and the accurate determination of the e-h quasi-Fermi level separation from measured spontaneous emission and amplified spontaneous emission spectra [17-19].

Methods. - The GaAs/AlGaAs heterostructures were grown by the metal-organic chemical-vapour deposition (MOCVD) technique. The active layer consisted of undoped $\mathrm{GaAs}$ and was grown on n-GaAs: Si [100] substrate. It was sandwiched by 2.0- $\mu \mathrm{m}$ thick $\mathrm{p}$ - and $\mathrm{n}$-type $\mathrm{Al}_{0.35} \mathrm{Ga}_{0.65} \mathrm{As}$ layers. The active layer was 0.1-0.2 $\mu \mathrm{m}$ thick. A 1.2- $\mu \mathrm{m}$ thick $\mathrm{p}^{+}-\mathrm{GaAs}$ layer was grown on top of the $\mathrm{p}-\mathrm{Al}_{0.35} \mathrm{Ga} \mathrm{G}_{0.65} \mathrm{As}$ layer. The top metal electrode had a 3-section configuration for a separate application of forward and reverse biases on the sections of the devices (see Fig. 2). Two 10- $\mu \mathrm{m}$ wide gaps were etched in $\mathrm{p}+-$ GaAs and in a part of $\mathrm{p}-\mathrm{Al}_{0.35} \mathrm{Ga}_{0.65} \mathrm{As}$ layers. This allows for the detection of spontaneous emission (SE) generated upwards in the active GaAs layer. The spontaneous emission was collected by a lensed fibre and analysed by an optical spectrum analyser.

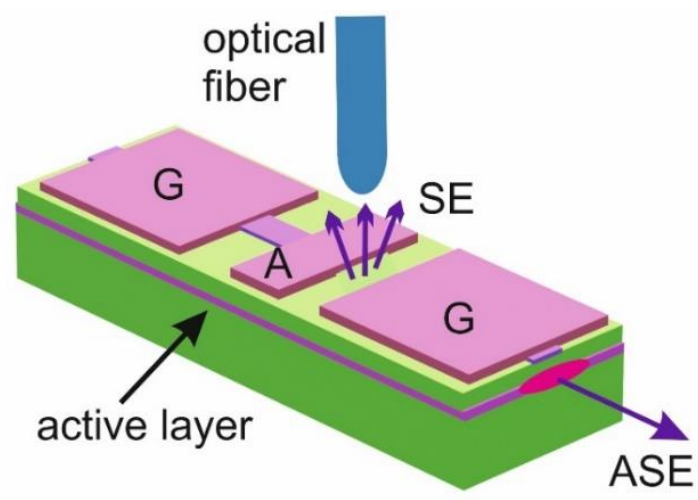

Fig. 2: A schematic of a heterostructure under test. $\mathrm{G}$ - two amplifying sections with a forward bias, A - an absorber section with a reverse bias. SE is spontaneous emission, ASE is amplified spontaneous emission

A characteristic feature of the structures is the presence of three sections along the axis of the device, two being optical amplifiers, while the other being an absorber. The two gain sections were normally connected and either a forward d.c. bias or pulsed current was applied to them. There exists a relatively poor quality resonator in the structures formed by the cleaved facets of the chip. The power reflectivity of each cleaved facet is round 0.32 . A $5-\mu \mathrm{m}$ wide mesa structure was etched in the upper highly doped $\mathrm{p}^{+}$-GaAs layer for preventing current leakage in the lateral direction. Lasing generally occurs in the devices at large enough driving currents due to a huge optical gain in the GaAs layer. The onset of lasing clamps the e-h density in the active layer at the threshold level, which is typically $(1.5-2.0) \times 10^{18} \mathrm{~cm}^{-3}$. The quantum degeneracy criterium is not met at these densities. To achieve higher e-h densities we frustrate lasing by the introduction of a controllable optical loss using a strong reverse bias on the central absorber section (the Franz-Keldysh effect). This technique allows us to get much greater carrier concentrations and achieve the onset of the superradiant (SR) phase transition [13]. If the absorber reverse bias (or the optical loss) is larger than required, the amplified spontaneous emission is generated from the heterostructure instead of giant femtosecond SR pulses [12,15]. SE and ASE are used for the accurate determination of e-h densities during the SR phase transition.

Experiment. - The amplifier sections of the devices were pumped by current pulses with an amplitude up to $800 \mathrm{~mA}$. The duration of the pulses was varied in the range from 9 to $20 \mathrm{~ns}$, 
while the repetition rate was changed from 0.5 to $2 \mathrm{MHz}$. The samples were mounted on copper heat sinks and their temperature was controlled with an accuracy of about \pm 0.01 ${ }^{\circ} \mathrm{C}$. The central section of the structures was connected to a variable source of dc bias. A reverse bias from 0 to $-10 \mathrm{~V}$ was applied to the absorber section. At first, the amplitude of the current pulses and the reverse bias was chosen for achieving the stable generation of SR pulses. Then, the absorber bias was slightly increased and an additional optical loss was introduced in the active layer. As a result, the photon-mediated e-h pairing does not occur due to a lack of the photons, the SR phase transition does not take place, and SE and ASE emission were detected from above and from the edge of the heterostructure, respectively $[13,15]$. Here, the e-h density in G-sections of the sample stays the same since the current pulse amplitude is not changed.
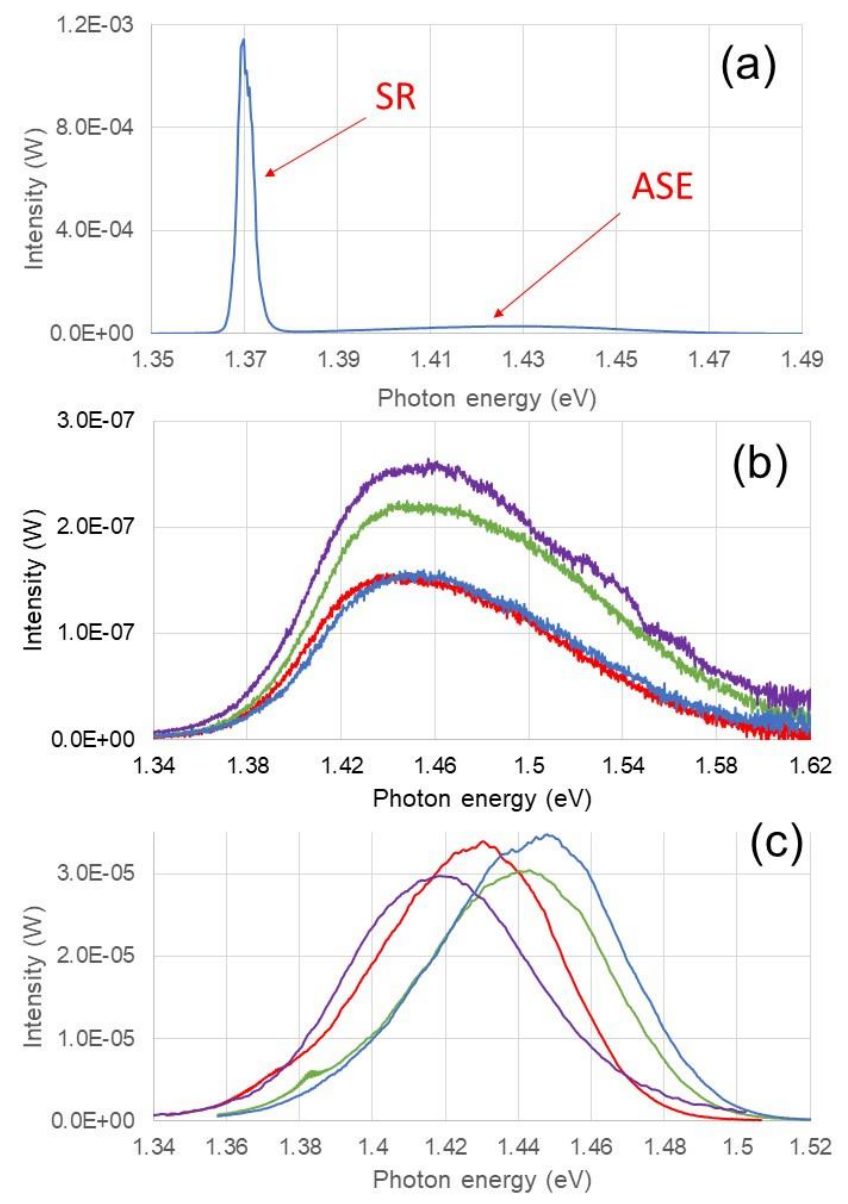

Fig. 3: Typical optical spectra of SR (a), SE (b) and ASE (c) detected from different GaAs/AlGaAs heterostructures.

Figure 3 presents the typical optical spectra of the SR emission, SE and ASE from different samples and at different driving conditions. The SR emission consists of a train of femtosecond pulses whose spectrum locates at the GaAs bandgap energy and has a minimum possible photon energy $[12,13]$. The femtosecond pulses are accompanied by nanosecond pulses of the ASE, which spectrum is located well above the band gap (Fig. 3 (a)). If the reverse bias on the absorber is increased by a fraction of volt, femtosecond SR pulses are not generated and one observes SE from the window at the top of the sample and ASE from the facet as presented in Fig. 3 (b) and (c), respectively. The onset of the SR phase transition in different samples occurs at different e-h densities above $5 \times 10^{18} \mathrm{~cm}^{-3}$. It is well-known that the band-gap shrinkage (renormalization) depends on e-h density [20-22]. That is why the position of the low-energy tail of both SE and ASE spectra varies on energy. The position of the peaks of the spectra is also changed depending on the e-h density and the parameters of heterostructures. For the estimation of the e-h density and e-h quasi-Fermi level separation, we use the measured renormalized bandgap energy $\tilde{E}_{\mathrm{g}}$ and parameters of the SE and ASE spectra as described below.

Calculations. - The criterium $\mathrm{n} \Lambda^{3}=1$ is fulfilled for pairs electrons-heavy holes in GaAs at $\mathrm{n} \cong 5.1 \times 10^{18} \mathrm{~cm}^{-3}$ at room temperature. For the estimation of the e-h density and e-h quasi-Fermi level separation at the SR phase transition, we employ the technique which has been previously described in detail [17-19]. The quasi-Fermi level separation is given by

$$
\Delta \mathrm{E}_{\mathrm{F}}=\hbar \omega_{\mathrm{p}}+\mathrm{kT} \ln \left(1+\frac{1}{\mathrm{R}^{\prime}}\right)
$$

where $\hbar \omega_{p}$ is the position of the optical gain peak and R' is approximately equal to

$$
R^{\prime} \cong k T\left\{\frac{1}{I_{S E}} \frac{d I_{S E}}{d \hbar \omega}-\frac{2}{\hbar \omega}\right\}_{\hbar \omega=\hbar \omega_{p}}
$$

There is the following relation of the derivatives of the spontaneous emission spectrum SE and the ASE spectrum on the photon energy at the optical gain peak [18]

$$
\left\{\frac{1}{I_{A S E}} \frac{d I_{A S E}}{d \hbar \omega}\right\}_{\hbar \omega=\hbar \omega_{p}}=\left\{\frac{1}{I_{S E}} \frac{d I_{S E}}{d \hbar \omega}\right\}_{\hbar \omega=\hbar \omega_{p}}
$$

Equation (4) implies that the position of the gain peak is exactly determined by the intersection of the two dispersion curves of the SE and ASE spectra for the given e-h density. Using Eqs. (2)-(3) and knowing $\hbar \omega_{\mathrm{p}}$ from Eq. (4), e-h quasiFermi level separation can be accurately calculated. The advantages of this approach are: (a) because the position of the gain peak is always lower than the peak positions of ASE and $\mathrm{SE}$, searching for $\hbar \omega_{\mathrm{p}}$ is done within only a narrow spectral region of ASE and SE below their peak positions, and (b) for determinations of $\Delta \mathrm{EF}_{\mathrm{F}}$ and $\hbar \omega_{\mathrm{p}}$ the measured SE and ASE spectra are required only without taking into account any other parameters that are difficult to obtain exactly [18]. Knowing $\Delta \mathrm{E}_{\mathrm{F}}$, the e-h density in the active GaAs layer can be found as follows. The density of electrons $n$ and holes $p$ is given by 


$$
\mathrm{n}=\mathrm{N}_{\mathrm{c}} \int_{0}^{\infty} \epsilon^{\frac{1}{2}} \mathrm{f}\left(\epsilon+\mathrm{E}_{\mathrm{c}}-\mathrm{E}_{\mathrm{Fn}}\right) \mathrm{d} \epsilon
$$

and

$$
\mathrm{p}=\mathrm{N}_{\mathrm{v}} \int_{0}^{\infty} \epsilon^{\frac{1}{2}} \mathrm{f}\left(\mathrm{E}_{\mathrm{Fp}}-\mathrm{E}_{\mathrm{v}}-\epsilon\right) \mathrm{d} \epsilon
$$

where the quasi-Fermi level separation of electrons and holes $\Delta E_{F}=E_{F n}-E_{F p}, \mathrm{f}(\epsilon)$ is the Fermi-Dirac function, and $\mathrm{N}_{c}$ and $\mathrm{N}_{\mathrm{v}}$ are the effective densities of states in the conduction and valence band, respectively. In addition, $n$ and $p$ are related by $n$ $=\mathrm{p}$ in the undoped GaAs layer. This relation and Eqs. (5) and (6) allow for calculating the e-h density at the SR phase transition.

The renormalized band gap energy $\tilde{E}_{\mathrm{g}}$ due to the band gap shrinkage induced by injected carriers can be found from the experimental SE and ASE using the method described in [22]. The band gap shrinkage depends on the e-h density. Both the experimental data and theory suggest that $\widetilde{E}_{\mathrm{g}}$ in bulk and MQW GaAs samples is proportional to $\mathrm{n}^{1 / \mathrm{m}}[21,22]$. The parameter $\mathrm{m}$ is in the range of from 2.6 [22] to 3.0 [21]. Knowing $\tilde{E}_{\mathrm{g}}$ from the experimental SE and ASE, one can estimate the e-h density and compare with theory. Figure 4 and 5 present the results of the processing of the experimental data. Since the quasi-Fermi level separation includes the value of the renormalized band gap $\tilde{E}_{\mathrm{g}}$, we estimate values of the e-h density which satisfy both measured parameters $\Delta \mathrm{E}_{\mathrm{F}}$ and $\Delta \tilde{E}_{\mathrm{g}}$ simultaneously. Figure 4 shows the estimated values of $\mathrm{n}$ at different $\Delta \tilde{E}_{\mathrm{g}}$ (blue circles).

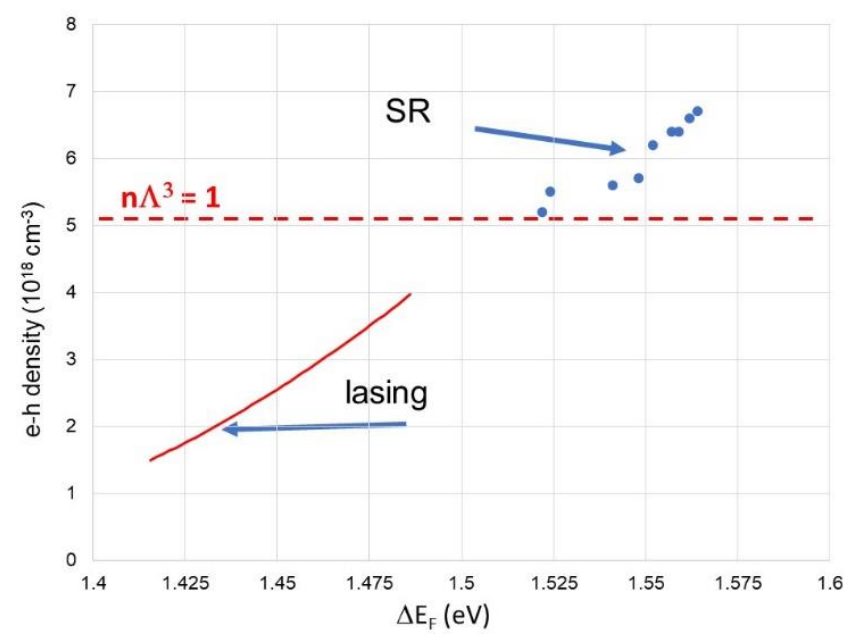

Fig. 4: E-h density vs. the quasi-Fermi level separation. The blue circles are the calculated data for SR from the measured SE and ASE spectra; the red line shows the calculated dependence from [23]

The e-h densities during the SR emission generation are in the range of (5.2-6.7) x $10^{18} \mathrm{~cm}^{-3}$, which correspond to the phasespace density $\mathrm{n} \Lambda^{3}$ of between 1.02 and 1.31. M.Yamada and H.Ishiguro calculated $\Delta \mathrm{E}_{\mathrm{F}}$ in undoped $\mathrm{GaAs}$ for $\mathrm{n}$ up to $4.0 \mathrm{x}$ $10^{18} \mathrm{~cm}^{-3}$ [23]. The red curve presents their theoretical results. The standard lasing in GaAs/AlGaAs heterostructures corresponds to the range of $\mathrm{n}=(1.5-2.0) \times 10^{18} \mathrm{~cm}^{-3}$, values of $\Delta \mathrm{E}_{\mathrm{F}}$ being by $100-150 \mathrm{meV}$ smaller as compared to $\mathrm{SR}$.
Figure 5 shows the measured values of the band gap shrinkage at different estimated values of $n$ (blue circles). The orange and blue curves show the theoretical results from [21] and [22], respectively. The minimum measured value of $\tilde{E}_{\mathrm{g}}$ is $1.371 \mathrm{eV}$. The typical SR spectrum locates right at the band gap energy as illustrated in Fig. 1 and as observed previously [12-15].

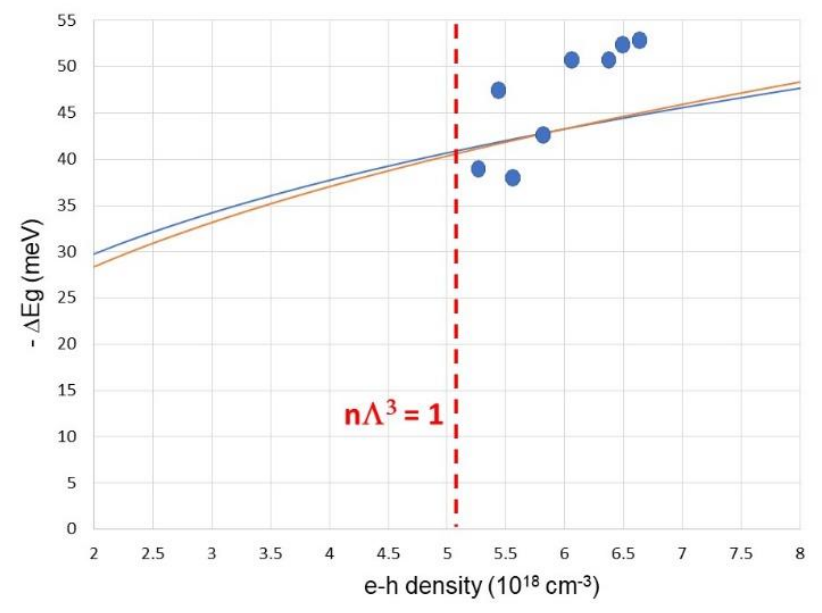

Fig. 5: Band gap shrinkage vs. e-h density. The blue circles are the estimated data for SR from the measured SE and ASE spectra; the orange and blue lines show the calculated dependence from [21] and [22].

Discussion. - The experimental results suggest that by contrast to lasing in $\mathrm{GaAs} / \mathrm{AlGaAs}$ heterostructures the quantum degeneracy criterium $n \Lambda^{3}>1$ is met during the SR emission generation. The maximum value of the estimated phase-space density in our experiments is 1.31 . This implies that wavefunctions of individual e-h pairs overlap a lot and form a macroscopic wavepacket. The build-up of a macroscopic polarization of ordered dipoles is a characteristic feature of the SR emission [13]. In an ideal case of low-density noninteracting bosons, the onset of Bose condensation occurs at a critical temperature given by

$$
T_{c}=(2 \pi / M)(n / 2.612)^{2 / 3}
$$

or at the critical phase-space density $\mathrm{n} \Lambda^{3} \cong 2.612[1,4]$. This value is twice as much as the measured phase-space density.

However, our experimental situation corresponds to a highdensity case $n a_{0}^{3} \gg 1$. Here the condensation may be viewed as Bose condensation of weakly bound electron-hole Cooper pairs, in much the same way as electron Cooper pairs condense in a superconductor $[24,25]$. As shown by Keldysh and Kopaev [6], anisotropic high-density electron-hole gas always undergoes an instability analogous to superconductivity. Electron-hole binding is then a cooperative effect rather than a single pair affair. In an ideal low-density Bose gas the critical temperature of condensation (Eq. (7)) is controlled by the centre-of-mass motion of bound pairs. In contrast, in a high- 
density case, $T_{c}$ is determined by pair breaking when bound pairs ionize. The BCS mean-field approach yields the following expression of $T_{c}$ [25]

$$
T_{c}=\left(\frac{e^{\gamma}}{\pi}\right) \Delta_{F}
$$

where $\gamma$ is Euler's constant and $\Delta_{\mathrm{F}}$ is the zero-temperature Fermi level gap. Eq. (8) gives a value of $T_{c}$ in bulk GaAs which is much lower than room temperature and is close to zero. Furthermore, the critical temperature drops as the phase space density increases [24]. For the explanation of the available experimental results, we proposed a possible mechanism and conditions of e-h condensation at room temperature [15].

We believe that resonant photons with energies at the band gap, which travel freely in the GaAs layer, establish coherence within the e-h ensemble and facilitate the build-up of a macroscopic wavepacket from overlapped wavefunctions of collectively paired electrons and holes. Here, we have photonmediated 'stimulated' condensation of e-h pairs in phase space at room temperature. As we noted above, the critical temperature in a high-density case is controlled by pair breaking. Due to very high e-h densities and strong degeneracy of the semiconductor in our experiment, a coherent BSC-like state of composite bosons (collectively paired electrons and holes) with the minimum possible energy exhibits quasi stability for a long enough time. There exists a broad subband within the conduction band which is full of unpaired electrons [15]. This subband prevents breaking of e-h pairs and loss of coherence and long-range order [14]. We determined the critical temperature of the condensate at a certain phase-space density by the condition of equality of the characteristic decay time of the e-h condensate to the characteristic incubation time of superradiance [9]. The developed theoretical model predicts $T_{c}$ of above $200 \mathrm{~K}$ at $\mathrm{n} \Lambda^{3} \sim 1$. The proposed approach explains all previously observed features of the SR phase transition, including a) the formation of the off-diagonal long-range order in the e-h system [14], which is typical for BEC; b) the approximation of SR spectra by Bogolubov quasiparticles instead of normal electrons and holes [12]; and c) the minimum possible energy of SR photons and anomalously strong spatial and temporal coherence of the SR emission [13-15].

The phase-space density can be obviously increased by decreasing the mass of e-h pairs $\mathrm{M}$ as it depends as $\mathrm{M}^{-3 / 2}$ (see Eq. (1)). We use the values of the effective electron mass of $0.067 \mathrm{~m}_{0}$ and the heavy hole mass of $\mathrm{m}_{\mathrm{hh}}=0.45 \mathrm{~m}_{0}$ for undoped bulk GaAs (mo is the free electron mass) [26]. It should be noted that the mass of heavy holes is different for $\mathrm{GaAs} / \mathrm{AlGaAs}$ heterostructures grown on substrates with different orientations. Indeed, $\mathrm{m}_{h}$ is equal to $(0.33-0.45) \mathrm{m}_{0}$ for the [100] substrate orientation and $\mathrm{m}_{\mathrm{hh}}=(0.60-0.77) \mathrm{m}_{0}$ for heterostructures grown on [111] substrates [27]. This means that it is more difficult to achieve quantum degeneracy in the latter case as compared to our heterostructures grown on [100] substrates. Moreover, effective masses of electrons and holes in GaAs depend on energy due to nonparabolicities of the conduction and valence bands [28]. Fortunately, both heavy and light hole masses increase with energy above the band gap and their values achieve the minimum right at the band gap, the location where the e-h condensation occurs during the SR phase transition.

Conclusion. - In summary, we have demonstrated that the quantum degeneracy criterium $n \Lambda^{3}>1$ can be fulfilled during the SR emission generation. The e-h density has been estimated by analysing both spontaneous emission from above the samples and amplified spontaneous emission radiated from their edges. This method has allowed for accurate determination of the e-h quasi-Fermi level separation and the band-gap shrinkage due to injected carriers. The maximum experimental value of the phase-space density is 1.31 . This value is not large enough for the onset of condensation within presently developed theories. It is supposed that resonant photons with energies at the band gap establish coherence within the e-h ensemble and facilitate the build-up of a macroscopic wavepacket from overlapped wavefunctions of collectively paired electrons and holes. As a result, the photonmediated 'stimulated' condensation of e-h pairs in phase space take place at room temperature. The presented technique of the evaluation of the phase-space density can be applied for another promising semiconductor heterostructures with low effective values of electron and hole masses, for instance for $\mathrm{AlGaAs} / \mathrm{InGaAs}$ heterostructures.

The authors acknowledge the funding from EPSRC (Grant \# EP/M013472/1). We would also like to thank H. Kan and H. Ohta for providing the samples for the present study.

\section{REFERENCES}

[1] Pethick C. J. AND SMITH H. Bose-Einstein Condensation in Dilute Gases (Cambridge University Press, Cambridge, UK), 2002.

[2] LegGett A.J. Rev. Mod. Phys. 73 (2001) 307.

[3] Cornell E. A. And Wieman C. E Rev. Mod. Phys. 74 (2002) 875.

[4] Griffin A., SNOKe D. W., AND Stringari S. C. (Editors), Bose-Einstein Condensation (Cambridge University Press, Cambridge) 1995.

[5] KASPRZAK J., RICHARD M., KUNDERMANN S., BAAS A., JeAmbrun J., KeEling J. M. J., Marchetti F. M., SZYMANSKa M. H., ANDRE R., StAeHLI J. L., SAVONA V.,. LitTlewood P. B, DEVEAUd B., AND DANG L.S. Nature (London) 443 (2006) 409.

[6] Keldysh L. V. And Kopaev Yu. V. Sov. Phys. Solid State 6 (1965) 2219.

[7] Comte C. And Mahler G. Phys. Rev. B 34 (1986) 7164.

[8] Cote R. And Griffin A. Phys. Rev. B 37 (1988) 4539.

[9] VASIL'EV P.P. And SMETANin I.V. Phys. Rev. B 74 (2006) 125206. 
[10] Murotani Y., Kim C., Akiyama H., Pfeiffer L. N., West K. W., AND ShIMANo R. Phys. Rev. Letts. 123 (2019) 197401.

[11] VASIL'EV P. Ultrafast Diode Lasers: Fundamentals and Applications (Artech House, Norwood) 1995.

[12] VASIL'EV P.P., KAN H., OHTA H., AND HiRUMA T. Phys. Rev. B 64 (2001) 195209.

[13] VASIL'EV P.P. Rep. Prog. Phys. 72 (2009) 076501.

[14] Vasil'Ev P.P., Olle V., Penty R.V., AND White I.H. Europhys. Lett. 104 (2013) 40003.

[15] VASIL'EV P.P. Phys. Stat. Solidi (b) 241 (2004) 1251.

[16] Vasil'ev P.P. And Penty R.V., New J. Phys. 22 (2020) 083046.

[17] Wu L. Appl. Phys. Lett. 76 (2000) 964.

[18] WU L. AND FU L. IEEE J. Quant. Electron. 36 (2000) 721.

[19] GuO W. H., Byrne D., Lu Q. Y., Phelan R., DONEGAN J. F., AND CORBETT B. IEEE Phot. Tech. Letts. 18 (2006) 1910.

[20] Vashishta P. And Kalia R. K. Phys. Rev. B 25 (1982) 6492.

[21] BENNETT B.R., SOREF R.A., AND DEL ALAMO J.A. IEEE J. Quant. Electron. 26 (1990) 113.

[22] TARUCHA S., KOBAYASHI H., HORIKOSHI Y., AND Окамото H. Jap. J. Appl. Phys. 23 (1984) 874.

[23] Yamada M.AND Ishiguro H. Jap. J. Appl. Phys. 20 (1981) 1279.

[24] Comte C. and Nozieres P. J. Phys. (Paris) 43 (1982) 1069.

[25] Nozieres P. AND ScHMiTT-RINK S. J. Low Temp. Phys. Rev. 59 (3-4) (1985) 195.

[26] Blakemore J.S. J. Appl. Phys. 53 (1982) R123.

[27] BingGeli N. ANd BALDERESCHI A. Phys. Rev. B 43 (1991) 14734.

[28] Lowney J.R. And Kahn A.H. J. Appl. Phys. 64 (1988) 447. 